\title{
Non-Linear Characteristics of the Photoinduced Deformation in Azobenzene-Containing Monolayers at the Air-Water Interface
}

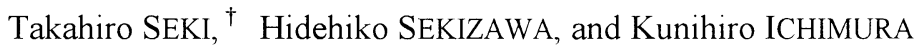

\author{
Photofunctional Chemistry Division, Research Laboratory of Resources Utilization, \\ Tokyo Institute of Technology, Nagatsuta, Midori-ku, Yokohama 226-8503, Japan
}

(Received January 7, 1999)

\begin{abstract}
Poly(vinyl alcohol)s having an azobenzene (Az) side chain (6Azn-PVA, $\mathrm{n}$ being the carbon number of the methylene spacer connecting PVA and Az unit) form a mechanically stable monomolecular film on a water surface. Alternate irradiation of UV $(365 \mathrm{~nm})$ and visible $(436 \mathrm{~nm})$ light induces large expansion and contraction of this monolayer in a reversible way. The photoinduced deformation of an isolated monolayer domain could be visualized using a Brewster angle microscope combined with a specially designed mobile mini trough. 6Azn-PVA with the longer spacers $(n=5$ and 10$)$ clearly exhibited a non-linear response in UV induced expansion with respect to the trans-to-cis photoisomerization proceeding with an induction time before starting expansion, whereas that of the shortest spacer $(n=1)$ did not show such a behavior. For 6Az10-PVA monolayer, intermittent UV light irradiation showed an forward (UV) and backward (dark) motion. The above non-linear deformation behavior can be the consequence of a cooperativity stemming from the self-assembling nature of the trans-Az side chains with the longer spacer.
\end{abstract}

KEY WORDS Azobenzene / Photoisomerization / Monomolecular Films / Photomechanical Effects

/ Non-linear Response /

Monomolecular films (monolayers) at the air water interface are a class of typical nano-organized assemblies which possess a few nanometers thickness. It is anticipated that such two dimensional molecular systems provides an ideal system for elucidation of molecular motions and characteristics involved in the process. Precise understandings of molecular motions at interfaces should be of great help for creation of new polymeric organized systems. ${ }^{1,2}$

Investigations on photomechanical response in monolayers started at the beginning of 1980s. ${ }^{3,4}$ Ever since several groups extensively studied the photoresponse of various photochromic monolayers. ${ }^{3-10}$ However, the methods for studying photomechanical effects in monolayers are mostly limited to a macroscopic handling using a Langmuir film balance. As the other extreme, the information at a molecular (nano) scale is obtainable by absorption spectroscopy and surface potential measurements. With these in mind, our recent attention has been paid to microscopic observations which cover the ranges between the above scale levels. ${ }^{11-13}$ As illustrated in Figure 1, macroscopic observations with polarized microscope (Brewster angle microscope, BAM) ${ }^{11,12}$ and atomic force microscope (AFM) ${ }^{13}$ satisfactorily bridge the large scale gap between the macro- and nano-scales. ${ }^{14}$

We reported in 1993 that the monolayers of poly(vinyl alcohol) derivatives having an azobenzene side chain (6Azn-PVA, Figure 2) at the air-water interface show a large photoinduced deformation upon alternate UV and visible light illumination. ${ }^{8}$ The trans-to-cis transformation of $\mathrm{Az}$ induced by UV light $(365 \mathrm{~nm})$ illumination leads to a large increase in the dipole moment of this unit $(0.5 \mathrm{D}$ (trans) $\rightarrow 3.1$ (cis) for unsubstituted $A z^{15}$ ), and the $\mathrm{Az}$ moiety contacts with the water surface at low pressures This gives rise to a large expansion of the molecular film On visible light $(436 \mathrm{~nm})$ irradiation, the reverse process

\footnotetext{
${ }^{\dagger}$ To whom correspondence should be addressed.
}

takes place. These processes can be repeated with full reproducibility. The schematic illustration of the film motion is shown in Figure 3. The surface pressure-area isotherm, ${ }^{8-10} \mathrm{BAM}^{11,12} \mathrm{AFM}^{13}$ observation on mica, surface potential measurement ${ }^{12}$, and $\mathrm{X}$-ray reflectivity analysis $^{16}$ all support this mechanism.

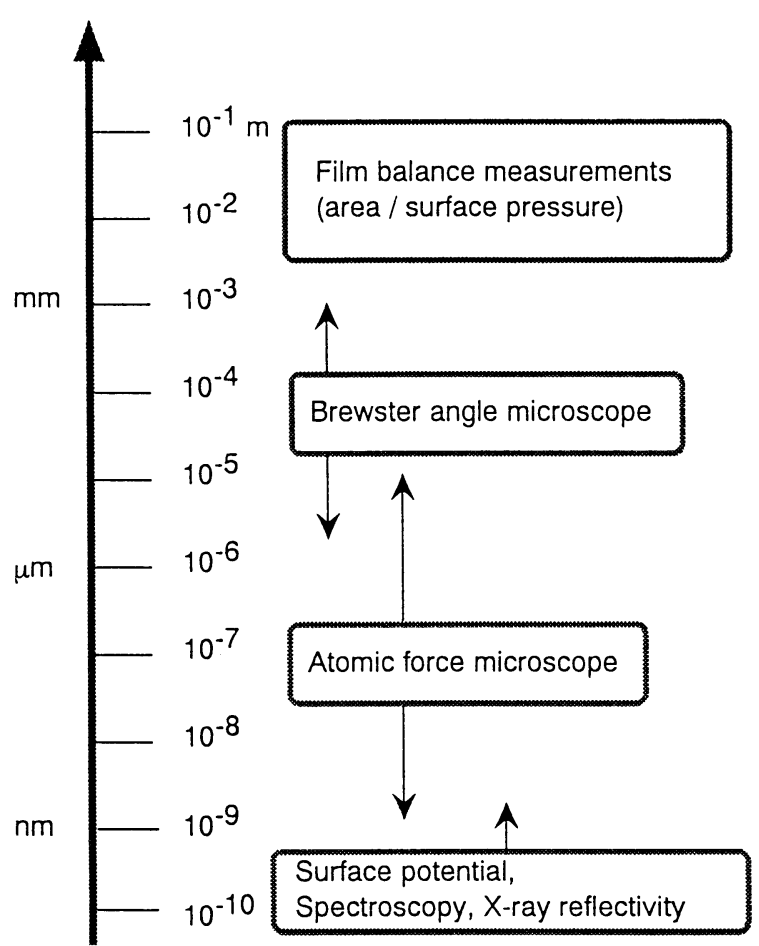

Figure 1. Tools for observation of photomechanical effects in monomolecular films arranged on the basis of typical covering length scales of observation. The X-ray reflectivity measurements is conducted by Yamaoka and Matsuoka at Kyoto University ${ }^{16}$ in the framework of the project of "New-polymers and their Nano-organized systems." 
It is stressed that the photoreaction of $\mathrm{Az}$ and motional vector of the individual side chain are aligned at the airwater interface maintaining the monolayer state. Therefore, the molecular event is exactly multiplied by the number of the floating side chains to yield an efficient and quantitative macroscopic deformation.

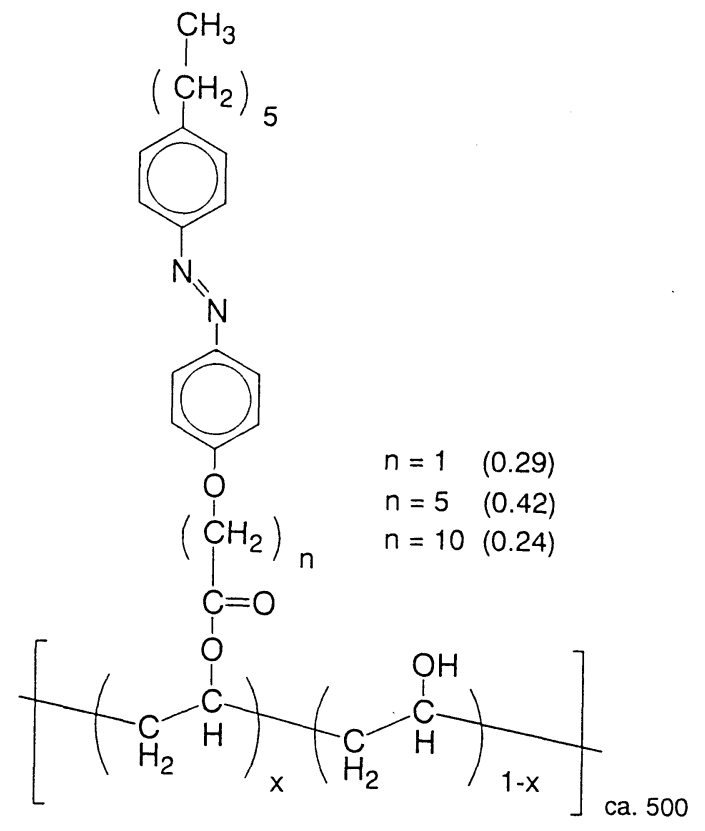

Figure 2. Molecular structure of 6Azn-PVA.
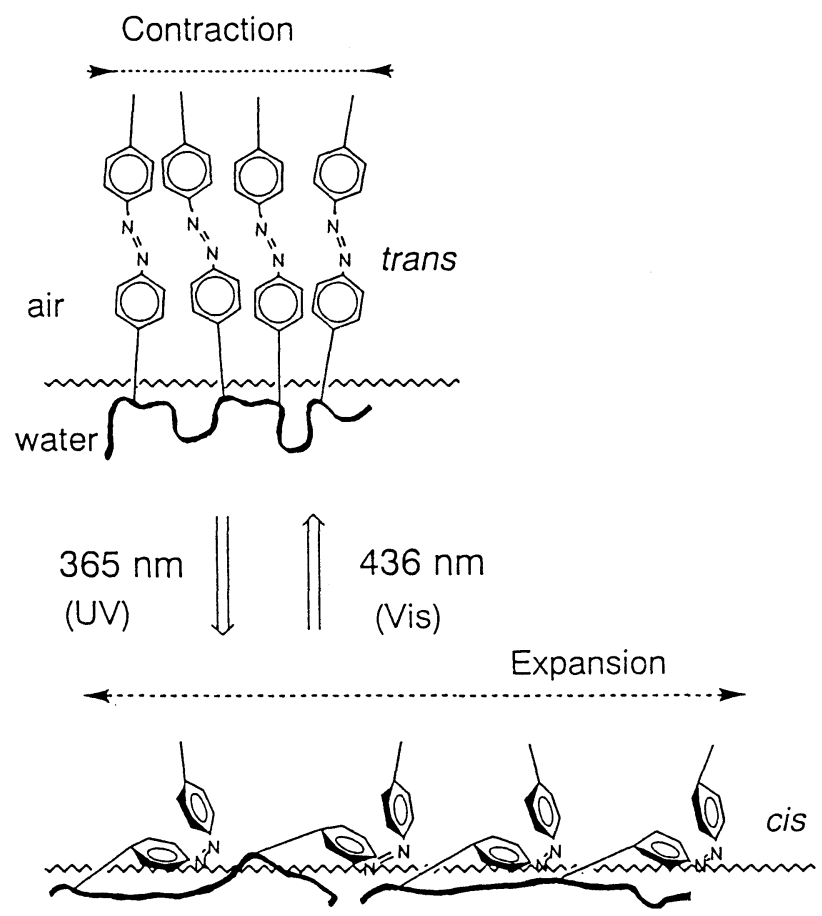

Figure 3. Schematic representation of photoinduced deformation of 6Azn-PVA monolayer on a water surface.

Our recent approach by BAM observation at zero pressure on the water surface revealed the cooperative nature of the photodeforming behavior in 6Az10-PVA monolayer. $^{12}$ The film expansion has been characterized by a non-linear response with respect to the photo- isomerization which involves an induction time before starting deformation.

This paper reports some results obtained in the extended study using 6Azn-PVA having the varied spacer lengths. The exploration with the structurally modified side chain was anticipated to give a deeper insight into this phenomenon.

\section{EXPERIMENTAL}

Synthesis of 6Azn-PVA was described in ref. 17

The monolayer was spread from a chloroform solution $\left(1.0 \times 10^{-3} \mathrm{~mol} \mathrm{dm}^{-3}\right)$ onto pure water (Milli-Q grade, 18 $\mathrm{M} \Omega \mathrm{cm}^{-1}$ ) filled in a Lauda FW1 film balance.

BAM images were taken with a NLE-EMM633 (Nippon Laser Electronics) equipped with a $10 \mathrm{~W} H \mathrm{He}-\mathrm{Ne}$ laser. The obtained images were recorded on a videotape and analyzed with the NIH image for the area estimation of monolayers. The trough used here was specially fabricated for monitoring a mobile isolated domain. A home-made mini rectangular trough $(3 \times 8 \mathrm{~cm})$ made of Teflon placed on an X-Y stage $(12 \times 12 \mathrm{~cm}$, Sigma Koki Co.) was used.

The UV-visible absorption spectra of monolayers at the air water interface were taken on a MCPD-2000 assembled with a deuterium/halogen lamp (Ohtsuka Electronics).

Light irradiation was performed with a $150 \mathrm{~W} \mathrm{Hg}$-Xe lamp (San-ei UV Supercure-203S). The wavelength selection was carried out by placing the combination of optical filters at the middle of the trough/illumination apparatus (see the photograph in Figure 4).

All procedures and measurements were carried out in dimmed red light at $20 \pm 1 \mathrm{C}$.

\section{RESULTS AND DISCUSSION}

Set up for Observation of Isolated Monolayer Domain

The isolated pressure-free domain of the photoresponsive monolayer was highly mobile, and it escaped rapidly from the microscopic field of BAM (diameter of ca. $0.6 \mathrm{~mm}$ ). For monitoring the area changes of such monolayer, a small trough placed on an $\mathrm{X}-\mathrm{Y}$ stage was fabricated. Figure 4 shows the photographic view of this set up. In this configuration, light irradiation was performed from the bottom of the trough through a quartz window (Figure 4, upper). The moving domain of monolayer could be monitored with sliding the $\mathrm{X}-\mathrm{Y}$ stage Additional great advantages of this configuration are that (i) the light intensity is accurately adjusted and calibrated, and (ii) if necessary (not undertaken in this work), polarized light illumination can be readily performed.

\section{Evaluation of Area Changes at Zero Pressure}

The spectroscopic evaluation revealed that the photoreaction itself initiated proceeding immediately on the illumination for the all cases (data not shown). The former report for 6Az10-PVA monolayer in comparison with the spectroscopic data ${ }^{12}$ demonstrated that the area stays constant up to a certain level of the trans-to-cis photoisomerization, and that the expansion starts with a delay. Thus, the photoinduced expansion is a non-linear process. We assume that this non-linearity can be a con- 

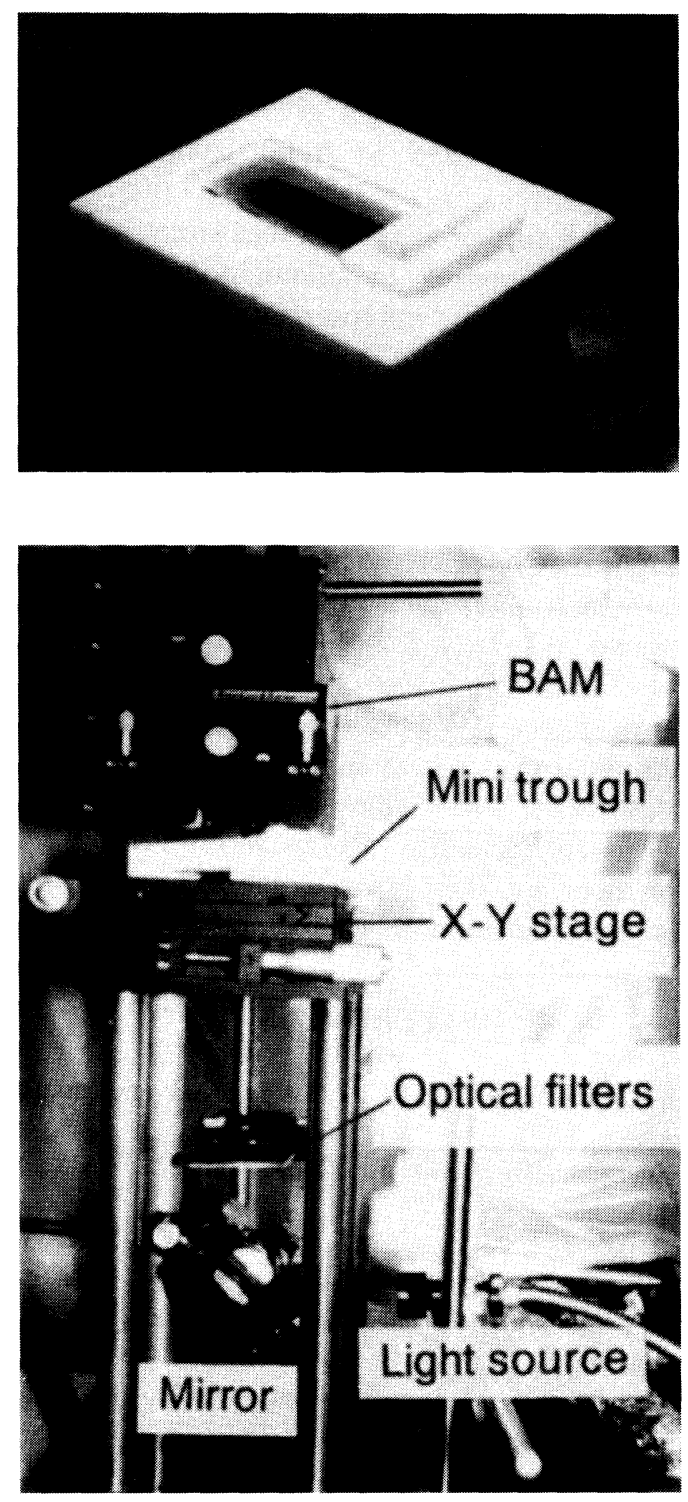

Figure 4. Photographs of the mini trough having a quartz window in the bottom (upper) and the apparatus for evaluation of area changes of an isolated monolayer (lower).

sequence of the competing actions of assemblage of the trans-Az side chains and expansion of cis-Az ones. It was anticipated that the 6Azn-PVA with varied spacer lengths modified the van der Waals attractive force among the trans- $\mathrm{Az}$ side chains in the monolayer to influence the photoresponsive behavior

Figure 5 displays the area changes of $6 \mathrm{Az} 1-\mathrm{PVA}, 6 \mathrm{Az} 5-$ PVA, and 6Az10-PVA monolayers as a function of irradiation time of UV light $\left(365 \mathrm{~nm}, 0.5 \mathrm{~mW} \mathrm{~cm}{ }^{-2}\right)$. The ordinate in this figure indicates relative areas to the initial one. For the three systems, the expansion commonly ceased almost in $200-300 \mathrm{~s}$ under the present conditions.

The magnitude of expansion which was estimated from the area after $250 \mathrm{~s}$ divided by the initial one were 1.6, 2.2, and 5.4 , for $\mathrm{n}=1,5$, and 10 , respectively. These figures are substantially larger, particularly for $\mathrm{n}=10$, than the previous macroscopic evaluations monitored at $2-3 \mathrm{mN} \mathrm{m}^{-1}$ with a Langmuir film balance ; 1,5 ( $=1), 1,9(\mathrm{n}=5)$, and $2.7(\mathrm{n}=10)^{8} \quad$ Obviously the BAM data at zero pressure Polym. J., Vol. 31, No. 11-2, 1999
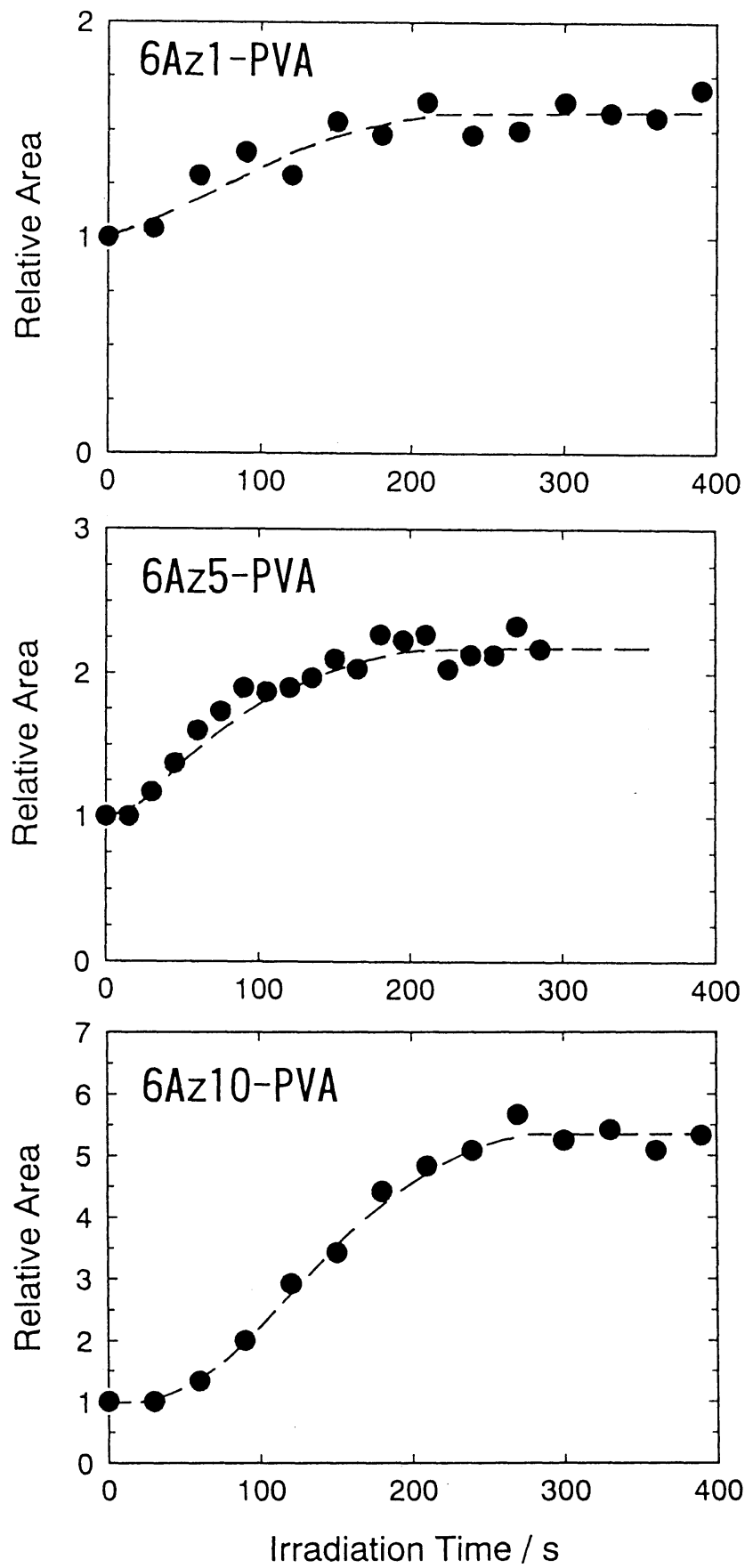

Figure 5. Relative area of 6Azn-PVA monolayer as a function of UV irradiation time. The light intensity was $0.5 \mathrm{~mW} \mathrm{~cm}$.

in this study provide the intrinsic expansion behavior, and the previous estimations contain compressed distortions stemming from the application of the surface pressure for area monitoring. As seen from the profiles, the induction period can be clearly recognized for 6Az10-PVA and 6Az5-PVA, whereas such non-linear behavior was hardly appreciated for 6Az1-PVA. For 6Az1-PVA monolayer, the magnitude of expansion was rather small for obtaining an accurate profile. Nevertheless, the difference in the profile feature is clear. The induction time observed for 6Az10-PVA monolayer was longer than that of 6Az5-PVA. Comparisons with the spectroscopic data revealed that the starting point of expansion was $10-20 \%$ and ca. $40 \%$ of the trans-to-cis conversions of $\mathrm{Az}$ for 6Az5-PVA and 6Az10-PVA monolayers, respectively. 
The self-contracting motion in the intermittent dark adaptation at earlier stages was observed for 6Az10-PVA (Figure 6). UV light illumination induced expansion of the film, on the other hand, the film showed a shrinkage immediately after the illumination was stopped. This is in consistence with the surface potential data under similar conditions. $^{12}$

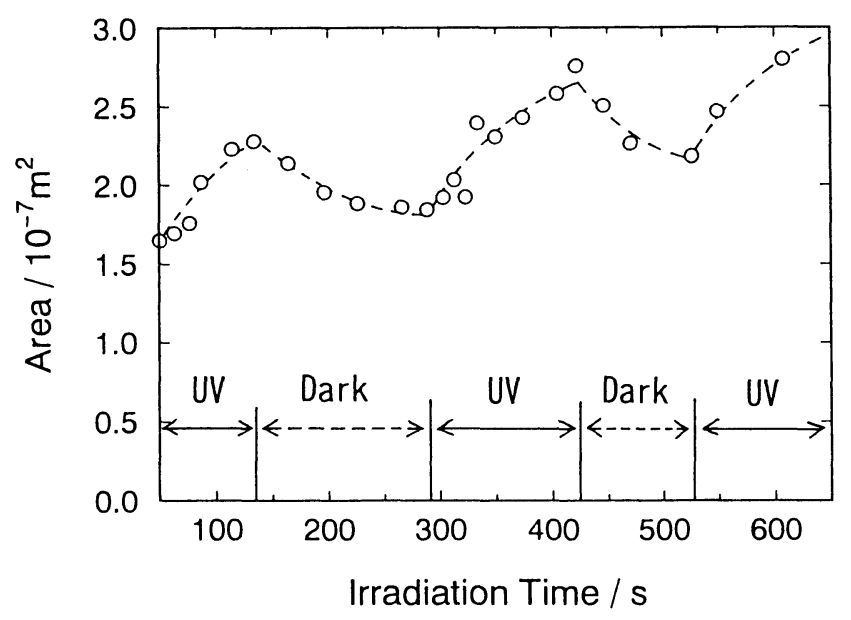

Figure 6. Changes in the area of 6Az10-PVA monolayer during the intermittent UV light irradiation at an early stage. The difference in the irradiation time from the data of Figure 5 is due to the variation of the exposure light intensity.

Our earlier paper described that the deformation of 6Az10-PVA monolayer when monitored at a low pressure stays without appreciable area changes at pauses of illumination (Fig. 2 of ref. 8). The contraction in the dark observed here seems somewhat inconsistent with the previous result. Our most recent paper described that, at 2 $\mathrm{mN} \mathrm{m}^{-1}$ the expansion starts to take place after most of the $\mathrm{Az}$ unit has been isomerized to the cis form. ${ }^{12}$ Thus, we observe the photoresponsive behavior at different stages of isomerization state between the present BAM observation at zero pressure and the previous area monitoring at $2 \mathrm{mN}$ $\mathrm{m}^{-1}$. The observed incongruity is attributable to this.

\section{CONCLUSION}

The previous ${ }^{12}$ and this work revealed the cooperative nature in the photoinduced expansion of the Az-side chain type polymer monolayer.

Variation of the spacer length connecting the main chain and the Az unit gave rise to a change in the time profile of the photoinduced deformation. The profile for monolayer having the longer spacer ( $n=5$ and 10) gave an obvious induction period before starting expansion. The monolayer of the shortest spacer $(n=1)$ hardly exhibited this behavior. These results should show that the selfassembling (contracting) action among the trans-Az side chains impede the immediate expansion at earlier stages, and that the expansion commences to occur after a certain amount of the cis-Az chain is accumulated. The van der Waals and $\pi$-stacking interactions can contribute to the contraction of the trans- $\mathrm{Az}$ side chains. The van der Waals force becomes stronger with the longer spacer material. This can lead to more distinct non-linear characteristics.

This approach further suggested that proper evaluations of mechanical responses in photochromic monolayers should be undertaken by microscopic observation at zero pressure together with the corresponding spectroscopic analysis. Precise understandings of mechanical response of polymer assemblies at a molecular level should be of particular help for designing and constructing new photoresponsive materials. The ultimate goal of this investigations may be to create hierarchical nano-organized structures which more closely resemble the biological motion system.

Acknowledgment. We thank S. Morino, M. Nakagawa, and $\mathrm{K}$. Arimitsu in our laboratory for technical assistance and helpful discussions. This work was supported by the Grant-in-Aid for Scientific Research on Priority Areas, "New Polymers and Their Nano-Organized Systems," from the Ministry of Education, Science, Sports and Culture.

\section{REFERENCES}

1. M. Irie, Adv. Polym. Sci., 94, 27 (1990). And references cited therein.

2. T. Kinoshita, Prog. Polym. Sci., 20, 527 (1995). And references cited therein.

3. H. S. Blair, H. I. Pogue, and J. E. Riordan, Polymer, 21, 1195 (1980).

4. H. Gruder, R. Vilanove, and F. Rondelez, Phys. Rev. Lett., 44, 590 (1980)

5. B. R. Malcolm and O. Pieroni, Biopolymers, 29, 1121 (1990).

6. H. Menzel, Macromol. Chem. Phys., 195, 3747 (1994).

7. M. Higuchi, M. Minoura, and T. Kinoshita, Colloid Polym. Sci., 273, 1022 (1995).

8. T. Seki and T. Tamaki, Chem. Lett. 1993, 1739

9. T. Seki, R. Fukuda, M. Yokoi, T. Tamaki, and K. Ichimura, Bull. Chem. Soc. Jpn., 69, 2375 (1996).

10. T. Seki, H. Sekizawa, R. Fukuda, T. Tamaki, M. Yokoi, and K Ichimura, Polym. J., 28, 613 (1996).

11. T. Seki, H. Sekizawa, and K. Ichimura, Polym. Commun., 38, 725 (1997)

12. T. Seki, H. Sekizawa, S. Morino, and K. Ichimura, J. Phys. Chem. B., 102, 5313 (1998).

13. T. Seki, K. Tanaka, and K. Ichimura, Macromolecules, 30, 6401 (1997).

14. T. Seki, H. Sekizawa, K. Tanaka, Y. Matsuzawa, and K Ichimura, Supramol. Sci., 5, 373 (1998).

15. D. J. Bullock, C. W. N. Cumper, and A. I. Vogel, J. Chem. Soc. $1965,5316$.

16. K. Kago, M. Feurst, H. Matsuoka, H. Yamaoka, and T. Seki, Langmuir, 15, 2237 (1999)

17. T. Seki, M. Sakuragi, Y. Kawanishi, Y. Suzuki, T. Tamaki, R. Fukuda, and K. Ichimura, Langmuir, 9, 211 (1993). 